\title{
Charge-independence-breaking in the triton
}

\author{
W. Glöckle, T.-S. H. Lee, and R. B. Wiringa
}

Physics Diyision. Argonne National Laboratory. Argonne. IL 60439

\begin{abstract}
We find the effect of the observed charge-independence-breaking in ${ }^{1} S_{0}$ nucleonnucleon scattering on the binding energy of the triton to be of order $80 \mathrm{keV}$. When corrections for this effect are made in an 18-channel momentum-space Faddeev calculation, we fird the Paris and Argonne $v_{14}$ potentials give triton binding energies that differ by only $20 \mathrm{keV}$.
\end{abstract}

\section{DISCLAIMER}

This report was prepared as an account of work sponsored by an agency of the United States Government. Neither the United States Government nor any agency thereof, nor any of their employees, makes any wartanty, express or implied, or assumes any legal liability or responsibility for the accuracy, completeness, or usefulness of any information, apparatus, $p$ oduct, or process disclosed, or represents that its use would not infringe privately owned rights. Refer. ence herein to any specific commercial product, process, or service by trade name, trademark, manufacturer, or otherwise does not necessarily constitute or imply its endorsement, recommendation, or favoring by the United States Government or any agency thereof. The views and opinions of authors expressed herein do not necessarily state or reflect those of the United States Government of any agency thereof. 
In this short note we report a calculation of the charge-independence-breaking (CIB) effect in the triton. We assume no charge-symmetry-breaking (CSB) so $V_{n n}=V_{p p} \neq V_{n p}$. We start with the observation that the Paris potential ${ }^{1}$ and the Argonne $v_{14}$ potential $^{2}$ give very similar highquality fits ( ) low-energy nucleon-nucleon $(\mathrm{NN})$ scattering data and deuteron properties. The main difference between the two models is that the Paris potential is fit to pp data in $T=1$ states, while the Argonne $v_{14}$ potential is fit to np data. This difference is most noticeable in the ${ }^{1} S_{0}$ channel, and is illustrated in Table I where we compare the singlet scattering lengths of the two models with experiment. ${ }^{3}$ We exploit this difference to calcuiate the CIB effect in the triton.

Previous results for $\mathrm{CIB}$ in the triton with realistic interactions give differences of order $300 \mathrm{keV}$. Brandenburg, Sauer, and Machleidt ${ }^{4}$ made 5-channel momentum-space Faddeev calculations for various combinations of the one-boson exhange potentials HM1 (fit to np data) and EHM (fit to pp data), and the Reid soft-core potential (also fit to pp data). Friar, Gibson, and Payne $^{5}$ made 5-channel configuration-space Faddeev calculations for the Reid potential and a variant in which the repuslion of the ${ }^{1} S_{0}$ channel was altered to fit the np scattering length. These calculations give $300 \mathrm{keV}$ and $310 \mathrm{keV}$, respectively, as the difference between models where the ${ }^{1} S_{0}$ channel is fit to either pp or np data.

The present calculations have been made using the momentum-space Faddeev method developed at Böchum. 6 Our results for the Paris and Argonne $v_{14}$ potentials without CIB effects are shown in Table $I$ for 5- and 18-channel calculations. (The 18-channel binding energy we report here is $70 \mathrm{keV}$ greater than that ruported in Ref. 6.) The energies are in good agreement with those of other groups as shown. ${ }^{7}$ The Argonne $v_{14}$ potential gives $170 \mathrm{keV}$ more binding than the Paris potential in the 5-channel calculation, and $240 \mathrm{keV}$ more in the 18-channel calculation. The greater attraction of the Argonne $v_{14}$ model is due primarily to its more attractive ${ }^{1} S_{0}$ potential, as expected from the scattering length comparison. This is partially offset by the smaller deuteron D-state percentage in the Paris model.

A correct calculation of the three-nucleon ground state including $\mathrm{CIB}$, but neglecting $\mathrm{CSB}$, should use a potential fit to pp scattering data for the one pp or nn pair and a different potential fit 
to np data for the two np pairs. This can be done in the momentum-space Faddeev formalism by setting (for the triton)

$$
\mathrm{t}^{\mathrm{T}=1}=\frac{2}{3} \mathrm{t}_{\mathrm{nn}}^{\mathrm{T}=1}+\frac{1}{3} \mathrm{t}_{\mathrm{np}}^{\mathrm{T}=1}
$$

where $t_{\mathrm{NN}}^{\mathrm{T}=1}$ is the fully-off-shell $\mathrm{NN} t$-matrix calculated with the appropriate potential for the isospin triplet state. This form was suggested by Brandenburg, et al. ${ }^{8}$ It is a good approximation since the $T=3 / 2$ three-nucleon component which can be admixed with the $T=1 / 2$ component due to the presence of $\mathrm{CIB}$ forces is negligible. Friar, Gibson, and Payne used the same weighting to define the input potential for their configuration-space calculations of CIB. However, mixing the potentials would only yield our relation approximately.

In the present calculation we use Paris potential for $\mathrm{t}_{\mathrm{nn}}^{\mathrm{T}=1}$ and Argonne $\mathrm{v}_{14}$ for $\mathrm{t}_{\mathrm{np}}^{\mathrm{T}=1}$ to define our ${ }^{1} \mathrm{~S}_{0}$ interaction. The interactions in other partial waves are generated either from the Paris potential or from Argonne $v_{14}$. These results are shown in Table III. It is seen that the CIB effect makes the Paris result more attractive, and the Argonne result more repulsive, as expected. The difference between these two potentials becomes only $40 \mathrm{keV}$ in the 5-channel calculation, and only $20 \mathrm{keV}$ in the 18 -channel calculation.

The shift in energy from adding CIB is about $100 \mathrm{keV}$ in the 5-channel calculation, and about $80 \mathrm{keV}$ in the 18-channel calculation. Our 5-channel results are in substantial agreement with those of Refs. 4 and 5. The size of the effect seems to be fairly constant in the more precise 18channel calculation.

In summary, we have calculated the charge-independence-breaking effect due to $V_{n n}=V_{p p}$ $\neq \mathrm{V}_{\mathrm{np}}$ by using Eq. (1) to define a mixed ${ }^{1} \mathrm{~S}_{0}$ interaction from the Paris and Argonne $\mathrm{v}_{14}$ potentials. The calculated CIB effect is about $80 \mathrm{keV}$ in an 18 -channel calculation. The triton binding energies calculated from these two potentials are then very close to each other.

This work was supported by the U. S. Department of Energy, Nuclear Physics Division, under contract W-31-109-ENG-38. The calculations were performed at the National Magnetic Fusion Energy Computation Center, Livermore, California. 


\section{REFERENCES}

${ }^{1}$ M. Lacombe, B. Loiseau, J. M. Richard, R. Vinh Mau, J. Côté, P. Pirés, and R. de Tourreil, Phys. Rev. C21, 861 (1980).

2 R. B. Wiringa, R. A. Smith, and T. L. Ainsworth, Phys. Rev. C29, 1207 (1984).

3 O. Dumbrajs, R. Koch, H. Pilkuhn, G. C. Oades, H. Behrens, J. J. De Swart, and P. Kroll, Nucl. Phys. B216, 277 (1983).

${ }^{4}$ R. A. Brandenburg, P. U. Sauer, and R. Machleidt, Zeit. Phys. A 280, 93 (1977).

5 J. L. Friar, B. F. Gibson, and G. L. Payne, Phys. Rev. Cㄴ6 1140 (1987).

${ }^{6}$ A. Bömelburg, Phys. Rev. C34, 14 (1986), and references therein.

7 Ch. Hadjuk and P. U. Sauer, Nucl. Phys. A369, 321 (1981); T. Sasakawa and S. Ishikawa, Few-Body Syst. 1, 3 (1986); J. L. Friar, B. F. Gibson, and G. L. Payne, Phys. Rev. C37. 2869 (1988).

${ }^{8}$ R. A. Brandenburg, G. S. Chulick, Y. E. Kim, D. J. Klepacki, R. Machleidt, A. Picklesimer, and R. M. Thaler, Phys. Rev. C37, 781 (1988). 
TABLE I. Singlet NN scattering lengths in $\mathrm{fm}$.

\begin{tabular}{cccc}
\hline & $1_{\mathrm{a}_{\mathrm{nn}}}$ & $\mathrm{d}_{\mathrm{app}}$ & $1_{\mathrm{a}_{\mathrm{np}}}$ \\
\hline & $-18.45 \pm 0.46$ & $-17.9 \pm 0.2$ & $-23.748 \pm 0.010$ \\
experiment \\
Paris
\end{tabular}


TABLE II. Triton binding energies compared with other calculations.

\begin{tabular}{ccccc}
\hline & \multicolumn{2}{c}{ Paris } & \multicolumn{2}{c}{ Argonne v14 } \\
& 5-channel & 18-channel & 5-channel & 18-channel \\
& & & & \\
& 7.27 & 7.40 & 7.44 & 7.57 \\
present work & 7.30 & 7.38 & -1.44 & 7.57 \\
Hannover & 7.31 & 7.39 & 7.45 & 7.58 \\
Los Alamos-Iowa & 7.48 & 7.56 & & - \\
\hline
\end{tabular}


TABLE III. Triton binding energies with charge-independence-breaking effect in ${ }^{1} \mathrm{~S}_{0}$ channel: $t_{n n}$ is derived from Paris and $t_{n p}$ is derived from Argonne $v_{14}$.

Potential 5-channel 18-channel

\begin{tabular}{|c|c|c|}
\hline Paris & 7.27 & 7.40 \\
\hline${ }^{1} S_{0}\left(\frac{2}{3} t_{n n}+\frac{1}{3} t_{n p}\right)+\operatorname{Rest}($ Paris $)$ & 7.38 & 7.48 \\
\hline$(\Delta \mathrm{E})_{\mathrm{CIB}}$ & 0.11 & 0.08 \\
\hline Argonne $v_{14}$ & 7.44 & 7.57 \\
\hline${ }^{1} S_{0}\left(\frac{2}{3} t_{n n}+\frac{1}{3} t_{n p}\right)+\operatorname{Rest}\left(\right.$ Argonne $\left.v_{14}\right)$ & 7.34 & 7.50 \\
\hline$(\Delta E)_{C I B}$ & -0.10 & -0.07 \\
\hline
\end{tabular}



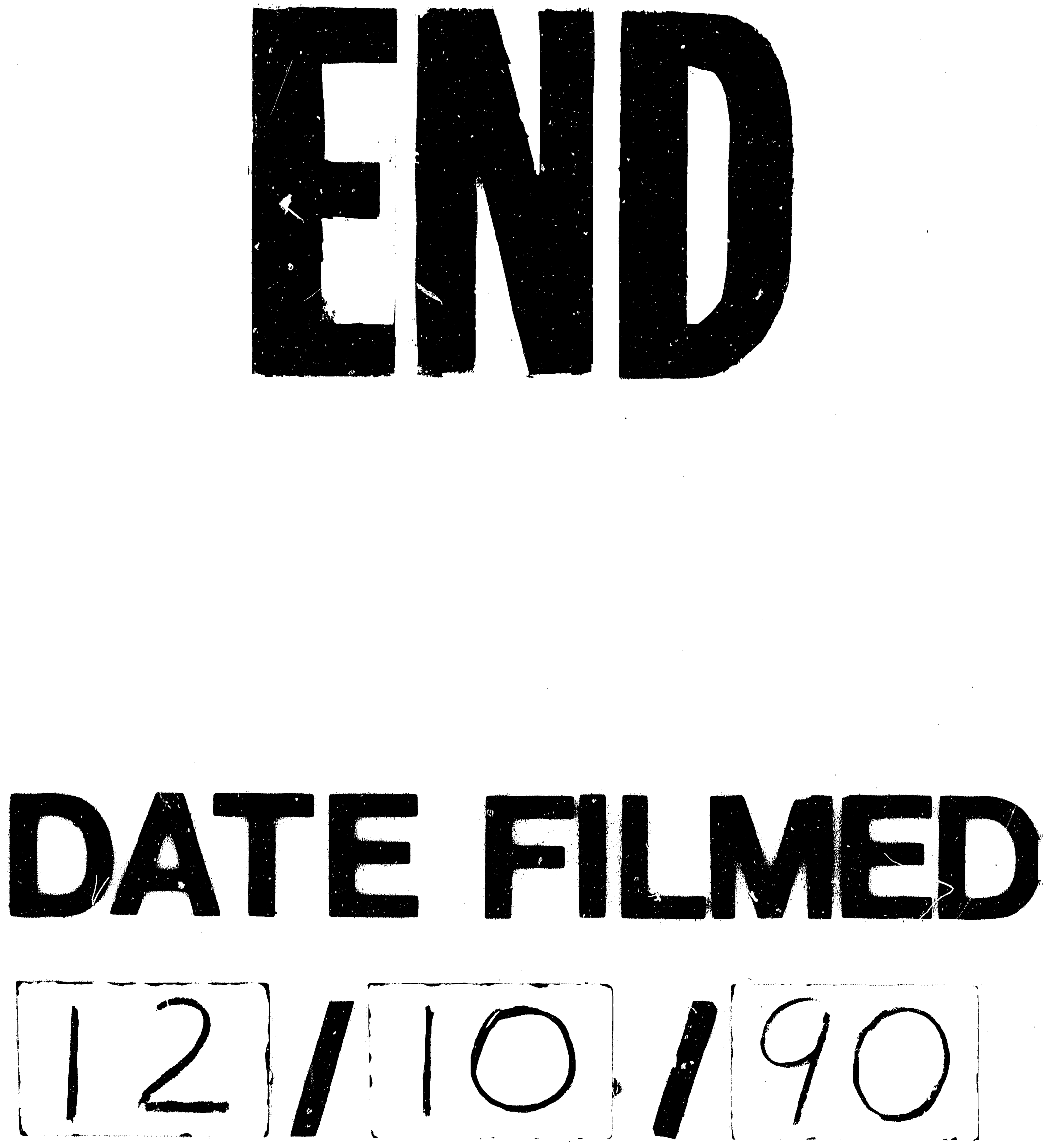
